of cement and binding of molecules. Supported structures on unstable soils should protect against this danger and therefore must be protected from this risk. These problems usually caused the erosion of the associated structure, tilt and fragmentation, Razouki et al. [7]. This type of land in Iraq comprises about $31.7 \%$ with gypsum levels between 10 and $70 \%$, Nashat [8].

Chen [9] examines the matter of mutual cooperation during a very straight sticky medium in small shear strains between multiple institutions. The basics are divided into different subcomponents of the examination. Dynamic response within each infrastructure is shown by the flexibleness of green. Dynamic impedance and consistency are integrated into the fundamentals by the limit of movement and also the balance of power of institutions. Broad results are implemented for unidentified circular founders placed in several sectors. Dynamic cooperation between neighbouring institutions and also the results on small number of institutions are firmly scattered.

The dynamic interaction of squared institutions under tight machine shaking was experimentally explored by Abhijeet and Priyanka in [10]. A series of thorough model tests during this field were conducted in dynamic conditions including an outsized range of isolated study and basic reactive response to local soil in Kanpur, India. The dynamic interaction of distinct groups (size) of two-legged clusters was examined by causing the vertical harmonic load to be continued one in each of the bases (active base), where the other footing (inert footing) had only a collection of weight. Dynamic interaction between active and passive bases was observed by investigating the transmission ratio variation $(T+)$ with the frequency ratio (fr). The passive base is resonant due to its vibrant excitement on the active base, which nevertheless occurs during a very phased delay of the resonant frequency of the active base. In determining the dynamic interaction of the baseline group, phase delay could also be a dynamic parameter. Phase delay is observed to be delayed when the spacing between surfaces decreases.

Chen [11] calculated the dynamic reaction by the numerical approach technique for a bunch of solid surface foundations. The formulation is completely stable and computationally simple with algebraic calculations. It does not impose any limitations on footing form, footing segmentation, layered medium thickness and frequency magnitude. Within the assessment. it is separated in several sub-square-regions from the foundation-base interface. By using the Green feature acquired through the transform and accurate Fourier-Bessel integration method, the dynamic reaction of each sub-region is calculated.

Sbartai [12] explored the dynamic interaction for two adjoining rigid foundations during a very viscoelastic layer. The vibrations come from one in each of the rigid soil layer foundations which have a harmonic load of translation, rocking and torsion. By taking into consideration their interaction, the dynamic responses to the rigid surface foundations are solved by the wave equations. The solution has been developed using the BEM (Bound Element) Frequency Domain technique. The evaluation of this research shows that the dynamic interactions between the two adjoining foundations have not had a negligible impact on multiple parameters. Particularly in respect to the other parameters, the dominant influence is clear just like the heterogeneity of the soil, the shape of the inspiration and also the load intensity.

Suraparb and Senjuntichai [13] investigated the dynamic response of two rigid foundations resting on a poroelastic medium with multilayered time-harmonic load. This research assumes soft, fully permeable contact surface between foundations and also the layered medium. A discretization method and an explicit stiffness matrix system are used to examine this dynamic interaction issue. They noted that the current solution's precision was checked for which numerical results were also presented for two square foundations impedance. They found that frequency of excitation and also the gap between the two bases were obviously tormented by the impedance features.

Han et al. [14] explored the dynamic interaction among two or more adjoining foundations on a laminated soil surface. Type of parametric studies was performed to clarify the implications of depth layer, soil dampening, spacing between adjacent foundation and support structural mass and moment of inertia and wave propagation velocity on the dynamic behaviour of the three-dimensional. Also the study provides numerical examples to assess the accuracy and computational stability of the proposed approach.

Ali et al. [15] determined the dynamic compressor foundation evaluation by using the ANSYS commercial finiteelement software. The model used a three-dimensional finite-element model. The impact of the underside foundation interaction during this study was taken into consideration within the model, which modelled soil as vertical and lateral spring and damper components. The soil foundation scheme determined basic natural frequencies and also the respective mode shapes and mass involvement ratios. The soil foundation reaction under forced excitation was calculated and displayed by the machine uneven load at distinct spanning frequencies.

In 2018, Vicencio and Nicholas [16] identified the effects of the structure-structure interaction (SSSI) between two buildings, with differing construction standards, distance and soil type. Using a nonlinear model (a simple twodimensional discrete), a set of nonlinear differential equations was defined. The results showed that both unfavourable and beneficial settings of the two structures generate 
a significant distinction between nonlinear SSSI and nonlinear SSI (non-separated construction state) of the soil just below the foundations. Linear rotational interaction is assumed between structures and linear behaviour of structures. The adverse effects of SSSI appear to be more pronounced when the behaviour of nonlinear soils is taken for granted.

Andersen [17] examined two polypod foundations dynamically interacted on the surface of the soil and two flexible embedded piles. In both cases, a layered ground with a soft top soil was considered over a rigid half-space. A Fortran effective implementation of a semianalytical approach was analysed in the frequency field by a soil foundation interaction using Green for a distributed load acting on a surface or an interface of a linear viscoelastic half-area with horizontal layers. The piles were modelled on the beams by finites and the soil at structure-soil-structure nodes, assuming the cross sections of the piles were rigid.

Keawsawasvong et al. [18] explored the dynamic response of rigid footing having arbitrary shapes on multilayer poroelastic half-space under time-harmonic moment loading by employing a way of discretionary process and a stiffness matrix approach. They founded when the gap from the neighbouring footing is twice greater than the width; rocking vibration is examined jointly footing. Rather, while the gap between these two footing increases, an unloaded foundation would still have radiated energy dissipated from a loaded foundation.

Therefore, the absence of empirical studies on the behaviour of the underside adjacent to the foundations of the machine encourages the study, explaining how two closely spaced institutions located on gypsum soil interfere dynamically with small models.

The urgent need for machinery and equipment within the event of cities is the main source of vibration that transports the soil and affects its engineering properties. The objectives of this work are to test the dynamic response of the muse to a sandy plaster soil under the influence of the combined dynamic load in both the states (dry and full) on the adjacent foundation. Dynamic answer includes displacement, vibration speed and acceleration.

This text provides an analysis of the dynamic response of one base with a tough and fast load near another base with a dynamic load, like (vibration source), in each of the foundations of a square shape, and thus, the opposite includes a circular shape, on gypsum soil. The study discusses the effect of the first foundation vibrations on the near foundation. On the soil surface, both institutions settle.

\section{Definition of problem}

There are two very far apart foundations, as shown in Fig. 1, on the gypsum soil. The load density belonging to the first base (vibration source) was maintained as $6 \mathrm{kN} /$ $\mathrm{m}^{2}$, while the second foundations were placed during a load range of $30 \mathrm{kN} / \mathrm{m}^{2}$ and thus the gap between the two establishments (S). The goal is to determine the dynamic responses of the second rule (acceleration of displacement amplitude) due to the dynamic excitement of application to the first rule both dry state and water.

\section{The material and method}

\subsection{Apparatuses of model}

Figure 2 shows the apparatuses of model; it includes the followings:
Fig. 1 Layout of experimental model

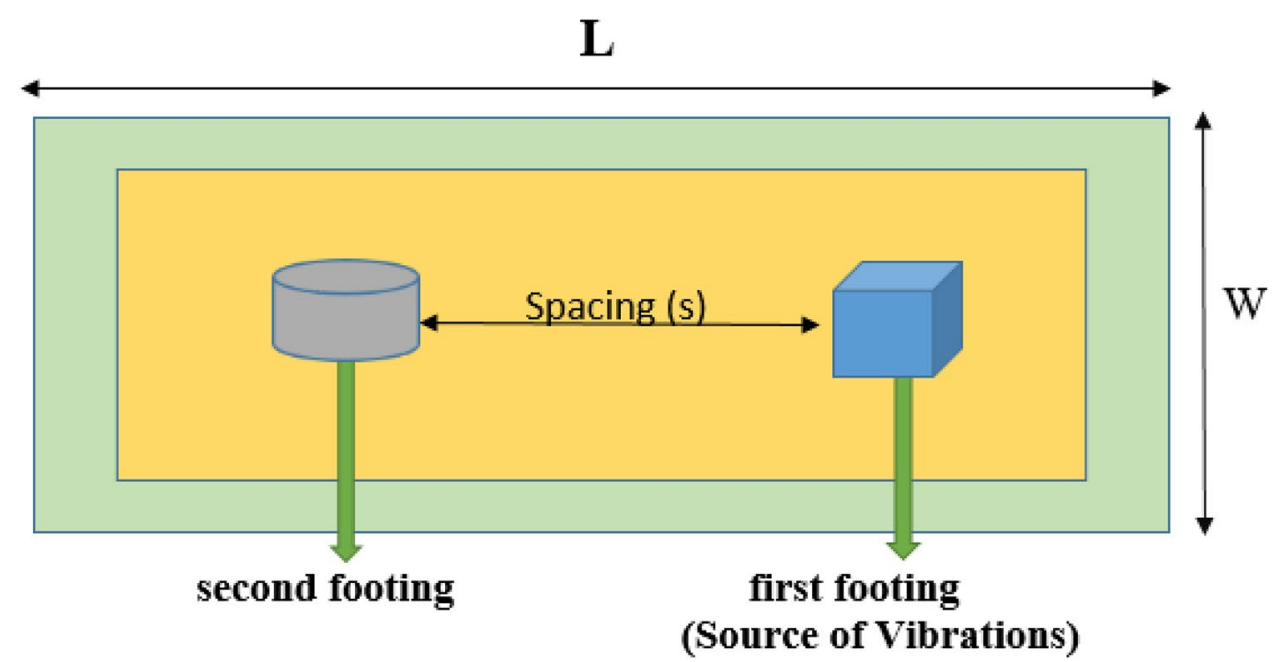

SN Applied Sciences A SPRINGER NATURE journal 


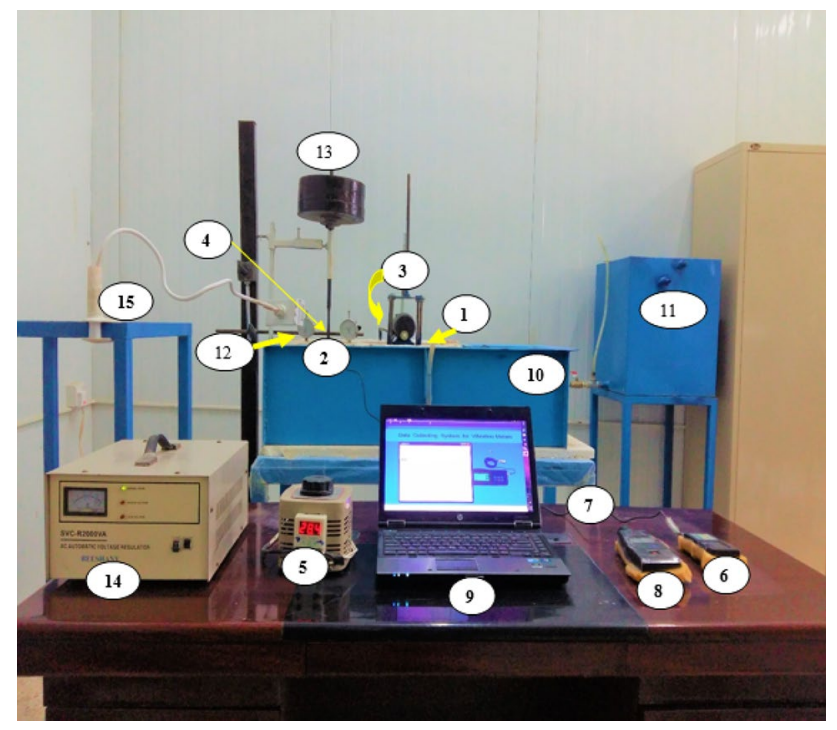

Fig. 2 The model with apparatuses

- Steel box with dimension $1000 \times 500 \times 500 \mathrm{~mm}$.

- Two foundations with dimension $80 \times 80 \times 40 \mathrm{~mm}$, for first foundation as a machine foundation, $80 \mathrm{~mm}$ in diameter, for second foundation which manufactured from steel

- Mechanical oscillator.

- Piezoelectric accelerometer.

- Two dial gauge.

- Variable frequency drive.

- Vibration meter.

- Digital tachometer.

- Computer device.

- Steel mould.

- Water tank.

- Static weight.

- Ac automatic voltage regulator.

- Camera

\subsection{Test Setup}

After examining previous studies by different researchers, like [19-24], a preliminary concept was developed; then, the experimental model group was designed. The primary concept could be a machine-based foundation with dimensions $80 \times 80 \times 40 \mathrm{~mm}$ (vibration source), and also the second base with $80 \mathrm{~mm}$ diameter is subject only to fixed load. Circular weights $20 \mathrm{~kg}$ with a diameter of $25 \mathrm{~cm}$ are accustomed steadily load the second footing, a mechanical oscillator of the rotating mass rather than the primary footing comes up with a varying dynamic load. The mechanical oscillator consists of steel turntables, with a diameter of $60 \mathrm{~mm}$ and a thickness of $13 \mathrm{~mm}$. With a tiny low eccentric block (I) instead of the turntable within the eccentric (e) of $15 \mathrm{~mm}$ from the axis of rotation. During this study, just one style of eccentric settings worth $50 \mathrm{~g}$ is employed. The DC motor is employed to convert the mechanical oscillator with a special frequency starting from $100 \mathrm{rpm}$ to $12,000 \mathrm{rpm}$. The controller is positioned outside the model to regulate the speed of the DC motor.

Before proving a dynamic response as displacement, speed or acceleration, the piezoelectric accelerometer is directly connected to the computerized model of digital vibration measurement (6063), which was previously defined by the computer. See Fig. 3.

The DT-2234A+ (digital tachometer) model was applied to guarantee that frequencies are not altered. See Fig. 4.

\subsection{Preparation and test procedure}

The soil for this study has been derived from the northern Iraqi Governorate, namely Tikrit, for the test programme; see Fig. 5 . The physical properties of the soil can be found in Table 1, and the chemical properties are shown

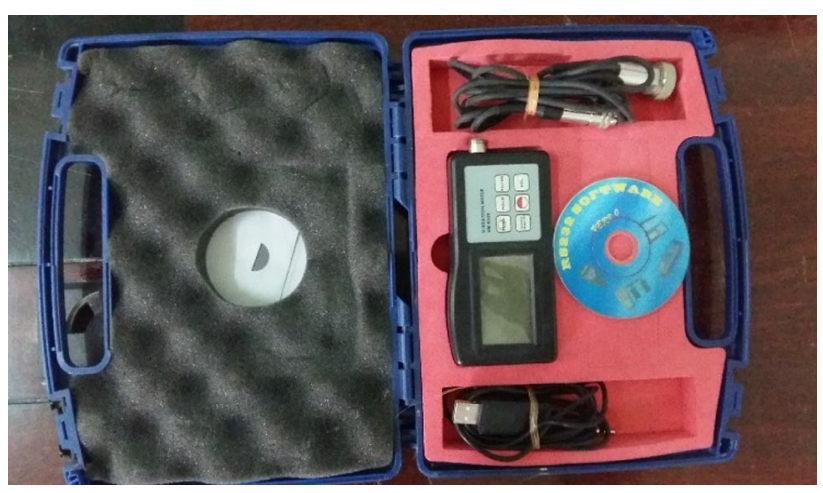

Fig. 3 Devices used for measuring vibration response

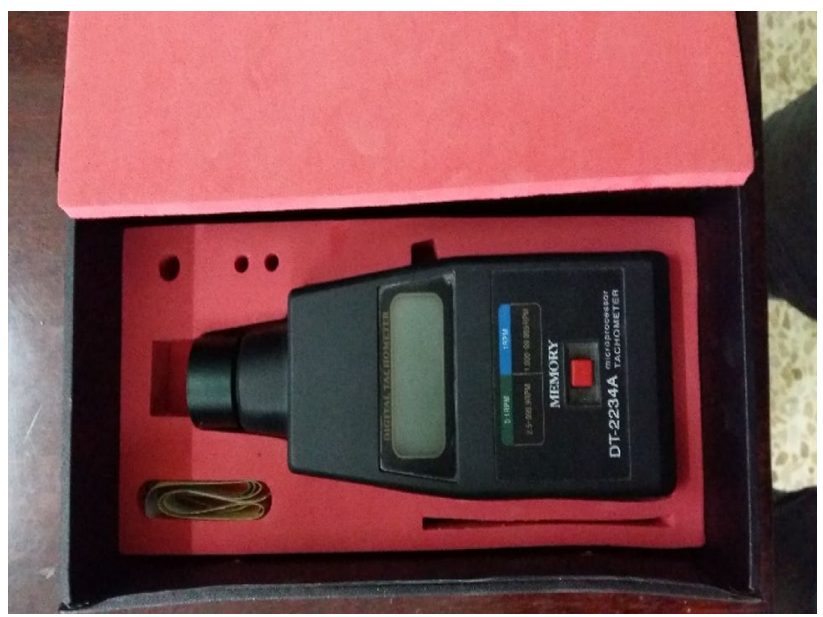

Fig. 4 Digital tachometer

\section{SN Applied Sciences}




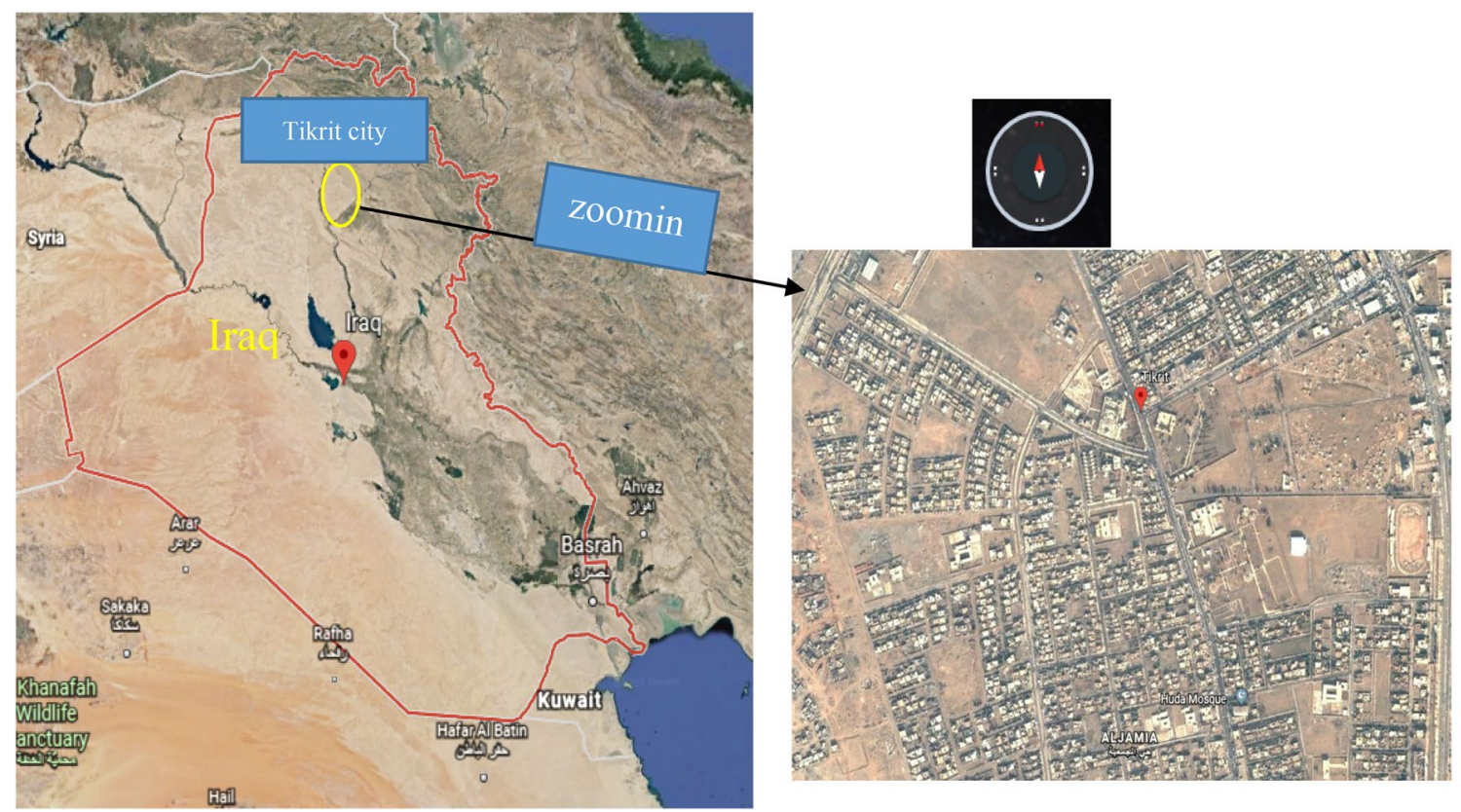

Fig. 5 The location of the soil sample (google earth image)

Table 1 Physical property of gypseous soil which is used for testing

\begin{tabular}{|c|c|c|c|}
\hline Test & Properties & Value & Specification \\
\hline & Specific gravity (Gs) & 2.41 & ASTM D 854 (2006) \\
\hline \multirow[t]{3}{*}{ Atterberg's limits } & Liquid limit (LL) \% & 21 & ASTM D4316-84 \\
\hline & Plastic limit (PL) \% & N.P & \\
\hline & Plasticity Index (PI) & N.P & \\
\hline \multirow[t]{4}{*}{ Compaction characteristics } & Compaction characteristics: & & ASTM 698-00 \\
\hline & Max. dry unit weight (KN/m3) & 16.23 & \\
\hline & Optimum moisture content \% & 12.33 & \\
\hline & Water content $\%$ & 2.8 & ASTM D2216-02 \\
\hline \multirow[t]{8}{*}{ Grain size analysis } & $\mathrm{D} 10(\mathrm{~mm})$ & 0.07 & ASTM D422-02 \\
\hline & $\mathrm{D} 30(\mathrm{~mm})$ & 0.14 & \\
\hline & $\mathrm{D} 60(\mathrm{~mm})$ & 0.35 & \\
\hline & Coefficient of uniformity, $\mathrm{Cu}$ & 5 & \\
\hline & Coefficient of curvature, Cc & 0.8 & \\
\hline & Passing sieve No. 200 (\%) (using kerosene) & 24 & \\
\hline & Classification of soil based on USCS & SM & \\
\hline & The collapse potential & 7.9 & ASTM D5533-2003 \\
\hline \multirow[t]{6}{*}{ Direct shear test } & Angle of internal friction $(\varnothing)$ in dry & 38 & ASTM D 3080-98 \\
\hline & Soil cohesion $(\mathrm{C})\left(\mathrm{KN} / \mathrm{mm}^{2}\right)$ in dry & 14 & \\
\hline & Angle of internal friction $(\varnothing)$ in soaked condition & 34 & \\
\hline & Soil cohesion $(\mathrm{C})\left(\mathrm{KN} / \mathrm{mm}^{2}\right)$ in soaked condition & 5 & \\
\hline & Test unit weight $\left(\mathrm{kN} / \mathrm{m}^{3}\right)$, $\gamma d$ test & 15 & \\
\hline & Field unit weight $\left(\left(\mathrm{kN} / \mathrm{m}^{3}\right)\right.$, field & 14.6 & ASTM D1556-07 \\
\hline
\end{tabular}

in Table 2. The results of a laboratory test performed on the sample soil used in the present research are shown in Figs. 6 and 7. Test water content at kept at $(45){ }^{\circ} \mathrm{C}$ to prevent loss of gypsum-soil crystal. The 65 per cent sample of gypsum soil is classified as (moderately severe) (ASTM D5533-2003). The gypsum soil (Sieve No. 4) has 
Table 2 Results of chemical properties of gypseous soil used for testing (BS 1377: 1990, Part 3)

\begin{tabular}{ll}
\hline Composition & Value \% \\
\hline Total soluble salts (TSS) \% & 67.2 \\
Gypsum content $\%$ & 65 \\
Sulphate content $\left(\mathrm{SO}_{3}\right) \%$ & 30.5 \\
Organic matters $(\mathrm{OM}) \%$ & 0.22 \\
Chloride content $(\mathrm{CL}) \%$ & 0.062 \\
$\mathrm{pH}$ value & 8.1 \\
\hline
\end{tabular}

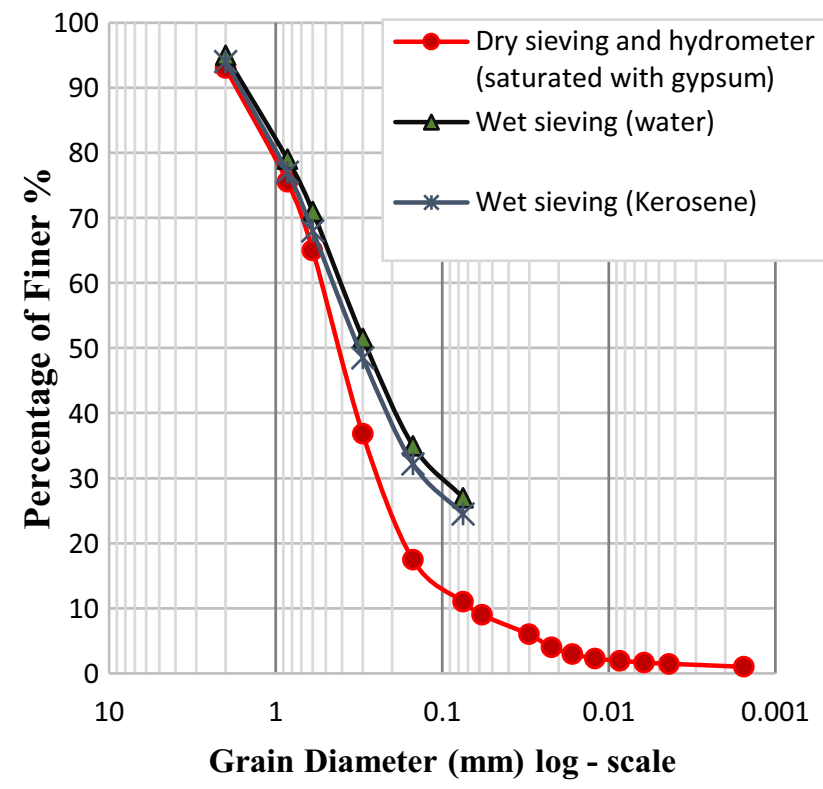

Fig. 6 Grain size distribution for gypseous soil

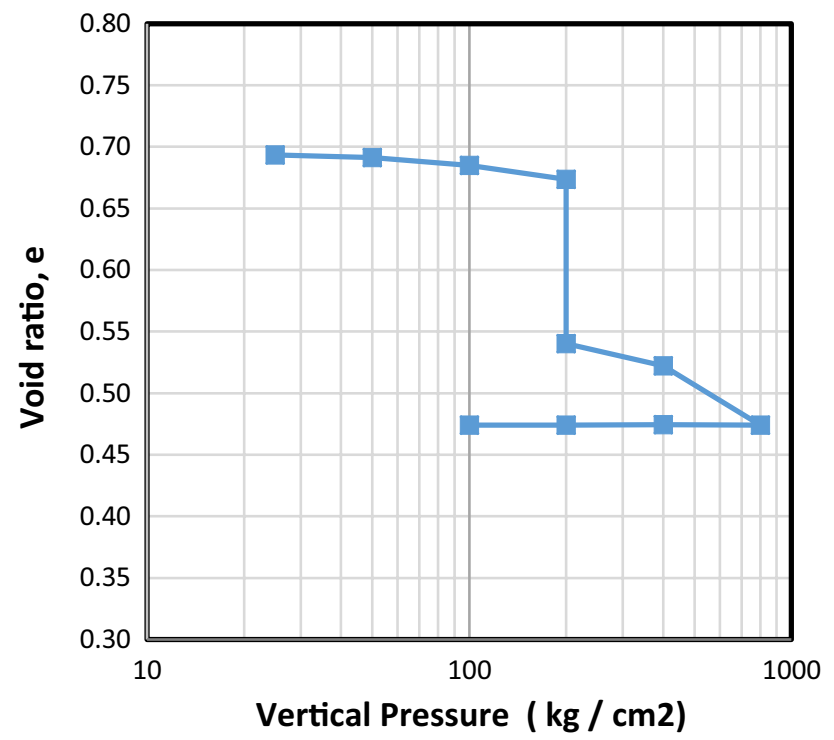

Fig. 7 Results of single oedometer been put in six layers of (steel container); with a uniform field density and by means of a hummer. A bubble ruler (equilibrium) system checks the surface and levels them. Both bases are centrally positioned over the prepared soil.

Figure 8 illustrates the test programme for this study.

The system's focus of gravity and the foundations must be kept vertically in line with the container's centre of gravity. After investigating results obtained by researchers and performing preliminary tests for $1-\mathrm{h}$ testing time, 30-min dry-zone operation test and 30-min soaking testing time, it should be noted that the steel container left for (24) $h$ for the soaking test to ensure that the soil was fully soaked, and in some cases soaked., and in the second day the test is continue.

In this study, the dynamic load is simulated with eccentric (me) setting $50 \mathrm{~g}$. The oscillator is then slowly operated by means of a velocity control unit, to avoid elevated dynamic load from occurring suddenly. The first foundation was therefore subjected to vertical vibration.

The dynamic reaction (displacement amplitude and acceleration) of the second base is evaluated and registered simultaneously using a Piezoelectrical accelerometer. The functioning frequency $(720,1080,1440)$ c.p.m. is regarded equal to $(12,18,24) \mathrm{Hz}$; the dynamic response parameters were recorded every five minutes during operation test duration.

\section{Results and discussion}

The present research examines the dynamic response of the circular footing with exciting dynamic force coming from an adjacent base as the basis of the machine.

In the second rule, dynamic analysis is performed by the first sensational rule with a vertical load density generated by the vibratory device after checking the stability of the rules under a fixed load. The displacement amplitude and acceleration of the second baseline are measured at different distances $(S=B, S=4 B, S=6 B)$, and for each state (dry and soaking), the two foundations are based on gypsum soil in the presence of the excitation dynamics applied at the first baseline. Figures 9, 10, 11 and 12 show the dynamic response of the second rule at separate distances to three frequencies $(12,18,24) \mathrm{Hz}$.

\subsection{Displacement capacity}

In figs. 9 and 10, maximum and minimum displacement capacity were plotted versus frequency for both dry condition and condition, and displacement amplitude increases at $(S=2 B)$ when the frequency of operation increases for both cases (dry and soaking). This increase is small if it ranges from 12 to $18 \mathrm{~Hz}$ and high if it reaches $24 \mathrm{~Hz}$ for 


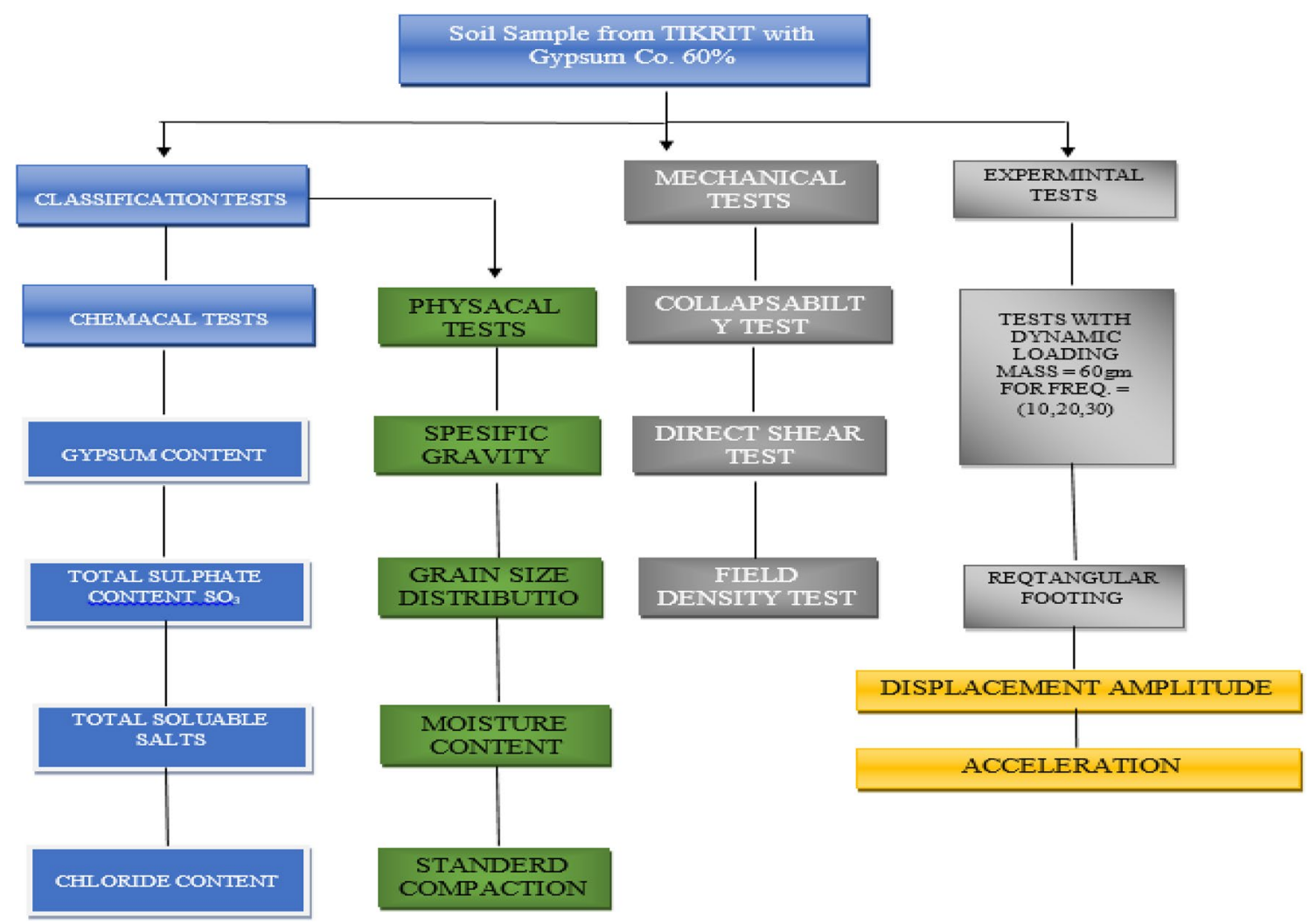

Fig. 8 Test programme

optimum capacity just in case of drought and sunken. In contrast, when $12-18 \mathrm{~Hz}$ or $24 \mathrm{~Hz}$ during a dry state, the minimum capacity indicates a small increase, but in the case of soaking, the worth doubles.

In soaking conditions, the displacement amplitude value decreases compared to the dry state of the three frequencies, due to the presence of water that acts as a wave inhibitor. During soil transfer when soaking, the vibrations from the first foundation were less energy and the displacement capacity of the second basis was reduced.

When the spacing is $4 \mathrm{~B}$, the displacement amplitude decreases compared to $(S=2 B)$ in both cases. The reason for this may be that the vibrations travel a protracted distance from the source vibration (first base) to the adjacent footing (second base). In other words, increasing distance lands up in reduced amplitude; the spread of vibrations across the soil ends up in reduced energy of those vibrations.

At the spacing of $6 \mathrm{~B}$, the number increased by half from 12 to $18 \mathrm{~Hz}$ to induce the foremost displacement capacity at drought and increased by 2.5 times in value at $24 \mathrm{~Hz}$. For the minimum displacement capacity, the price doubled from 12 to $18 \mathrm{~Hz}$ or $24 \mathrm{~Hz}$.

In soaking, the displacement capacity is reduced compared to the dry state. The utmost capacity is slightly higher when it ranges from 12 to $18 \mathrm{~Hz}$ but doubled when it rises to $24 \mathrm{~Hz}$. The minimum displacement capacity is different. The amplitude is doubled when it rises from 12 to $18 \mathrm{~Hz}$. But it increased by thrice the value of $24 \mathrm{~Hz}$. Here the displacement amplitude value decreased when comparing the amplitude volume at $(S=2 B)$ and $(S=4 B)$ for each case (soaked and dry).

\subsection{The acceleration}

The maximum and minimum acceleration vs. frequency are shown in Figs. 11 and 12 and are registered in a dry and soaked state for three spacing $(S=2 B, S=4 B, S=6 B)$. The direction of behaviour is similar in the case of dehydration and sunken for minimal and minimum accelerations. Acceleration increases with increasing frequency in dry and sunken conditions. The effects resulting from the increased distance between the two shots on the accelerated volume are comparable with the effect of displacement and increased distance that causes acceleration to decrease, because the proliferation of vibrations across the soil leads to a decrease in energy and thus a decrease in acceleration. We also note that the volume of acceleration is higher in the case of drought than in the case of soaking. 


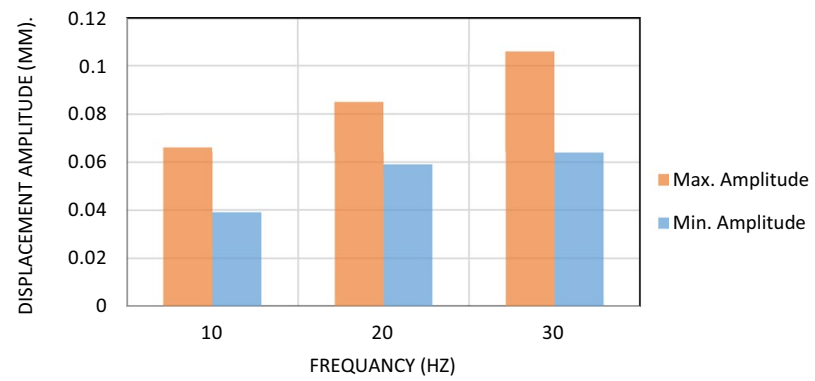

(a)

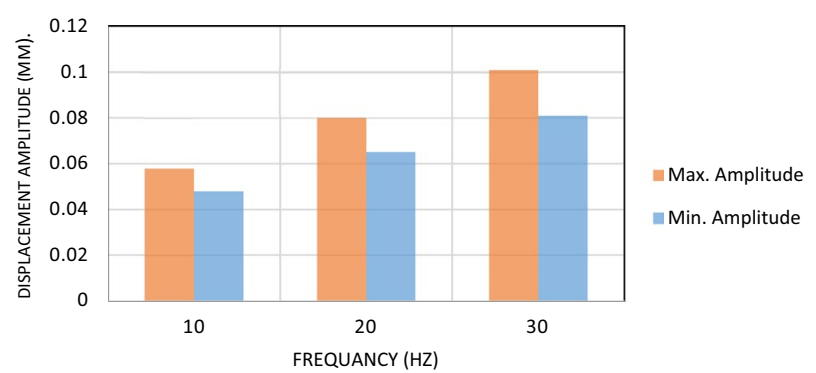

(b)

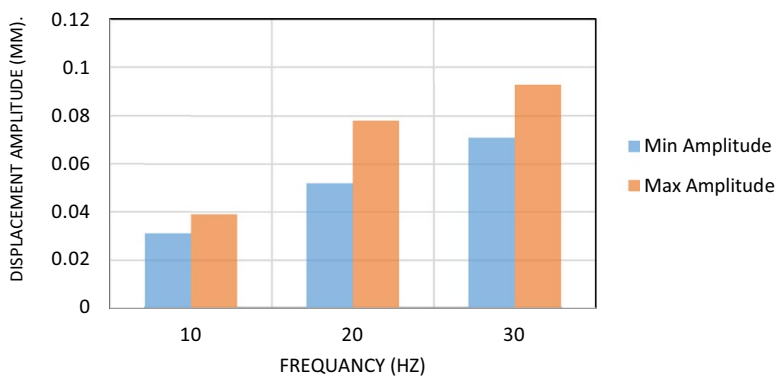

(c)

Fig. 9 The displacement amplitude versus frequency for different spacing (s), a at $S=2 B$, b at $S=4 B, c$ at $S=6 B$ ) for dry condition

The acceleration volume within the dry state increases almost linearly when the frequency increases from 12 to 18 $\mathrm{Hz}$ or $24 \mathrm{~Hz}$, at a distance (2B). The identical applies to (4B) and (6B) breaks. The gap between the utmost and also the minimum increases when the frequency rises and also the gap decreases when the gap between the two institutions increases as shown in Fig. 11.

In soaking condition, the acceleration rate increases only slightly for maximum and minimum accelerations when frequency increases from 12 to $18 \mathrm{~Hz}$, but is about double in value for $24 \mathrm{~Hz}$, which applies to the acceleration magnitude at a distance between the two footings as $4 \mathrm{~B}$, $4 \mathrm{~B}$ and $6 \mathrm{~B}$. When the frequency increases, the gap between the maximum and the minimum acceleration increases and decreases with increasing the distance between the two foundations. In comparison with their values at dry level, the acceleration values at soaked state for three frequencies 12,18 , and $24 \mathrm{~Hz}$, at a spacing of $2 \mathrm{~B}, 4 \mathrm{~B}$ and $6 B$, have fallen because of the presence of water, which

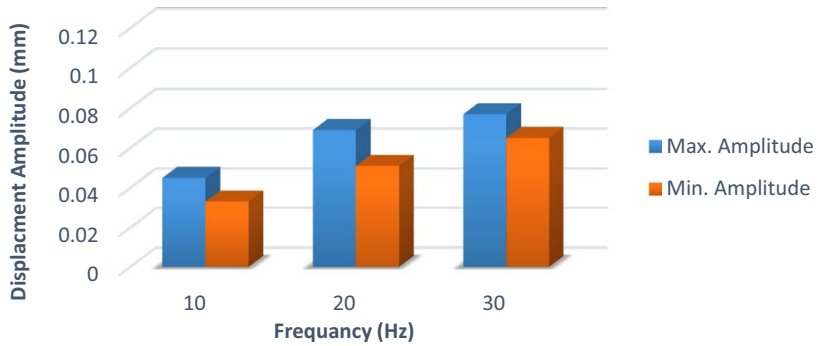

(a)

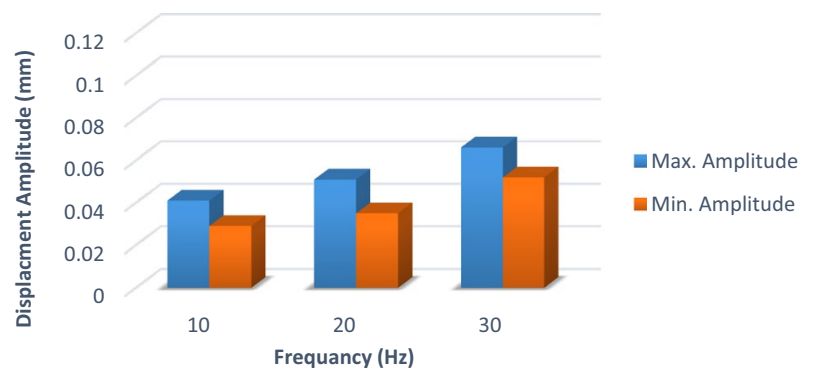

(b)

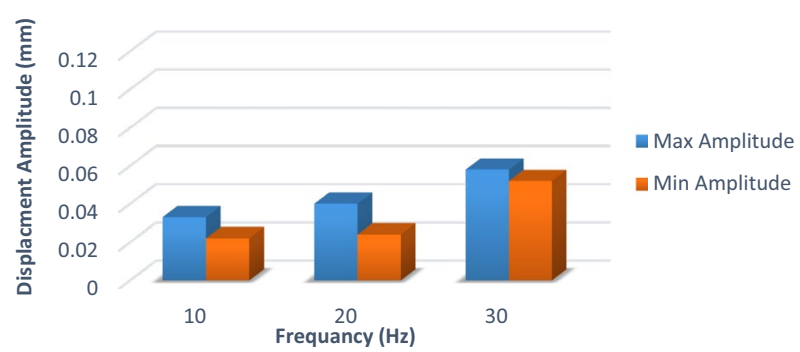

(c)

Fig. 10 The displacement amplitude versus frequency for different spacing (s), a at $S=2 B, b$ at $S=4 B, c$ at $S=6 B$ ) for soaking state

acts as a wave inhibitor in soil (as already mentioned) and increases when the frequency increases, irrespective of whether its state is wet or dry. When the distance between the two foundations increases, the acceleration is reduced for both soaked and dry conditions, see Fig. 12 .

\section{Conclusions}

\subsection{Displacement amplitude}

- The value of the displacement capacity of the footing under effects of dynamic loading comes from a close-by foundation (both footings are supported gypsum soil) with low operating frequency. The displacement in an undervalued state just in case of drought increases displacement capacity while reducing distance between feet. Reduction of displacement capacity when the space between the two foundations 
Fig. 11 The acceleration versus frequency for different spacing (s), a at $S=2 B, b$ at $S=4 B$, c at $S=6 \mathrm{~B})$ for dry condition

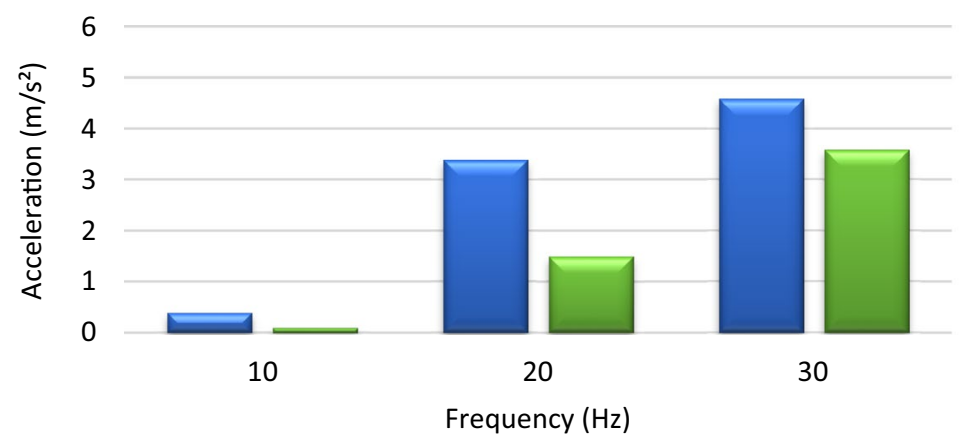

- Max. Acceleration $\square$ Min. Acceleration

(a)

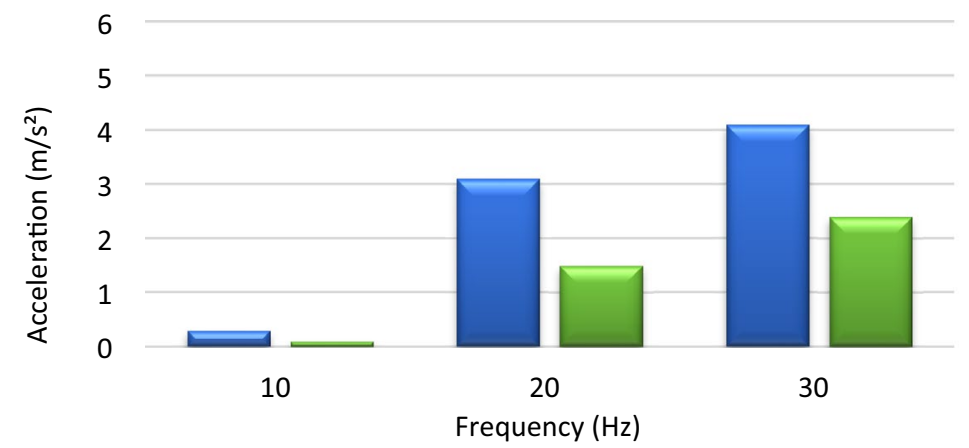

(b)

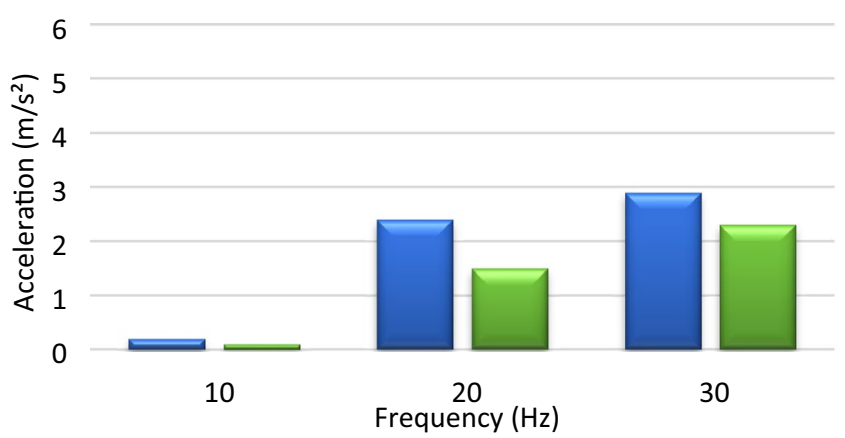

- Max acceleration

Min acceleration increases from $2 \mathrm{~B}$ to $4 \mathrm{~B}$ at a frequency of $12 \mathrm{~Hz}$ is $14 \%$ in the dry state and 7 in the case of soaking. When the space increases from $2 \mathrm{~B}$ to $6 \mathrm{~B}$, the reduction is $38.9 \%$ and $29 \%$ in the case of dehydration and soaking, respectively. At a frequency of $18 \mathrm{~Hz}$, the displacement capacity (6.5\% and $24.2 \%$ ) decreased in dry and sunken conditions, respectively, when the space increased from $2 \mathrm{~B}$ to $4 \mathrm{~B}$ and decreased by $9.2 \%$ and $40.9 \%$ just in case of drought and soaking, respectively, when the space increased from $2 B$ to $6 \mathrm{~B}$. For the frequency 24 $\mathrm{Hz}$, the reduction is $5.01 \%$ in the case of drought and $13.88 \%$ in the case of soaking when the space increases from $2 B$ to $4 B$. When the space increased from $2 B$ to $6 \mathrm{~B}$, the displacement capacity decreased $(11.96 \%$ and $25.1 \%)$ in dry and soaked conditions, respectively.
- There decrease in displacement capacity at dry condition when spacing increases from $2 \mathrm{~B}$ to $4 \mathrm{~B}$ or $6 \mathrm{~B}$ less than the decrease in soaking displacement capacity for the same spacing.

\subsection{Acceleration}

- The acceleration of the footing under the influence of dynamic loading resulting from the near foundation (on gypsum soil) increases with increasing operating frequency. The magnitude of acceleration in the dry conditions is greater than its value in the case of soaking.

- Acceleration increases with reduced distance between feet. Reduced acceleration when the gap between the 


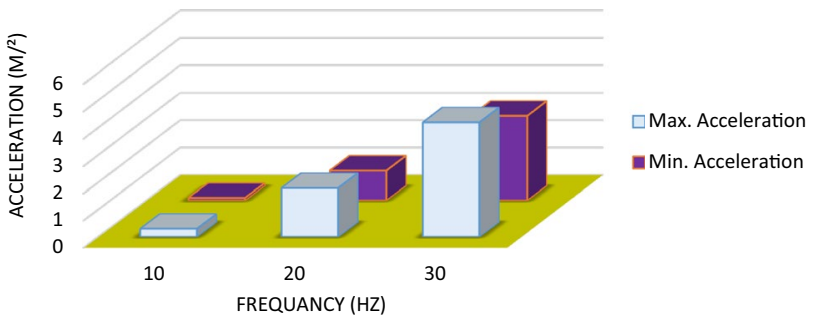

(a)

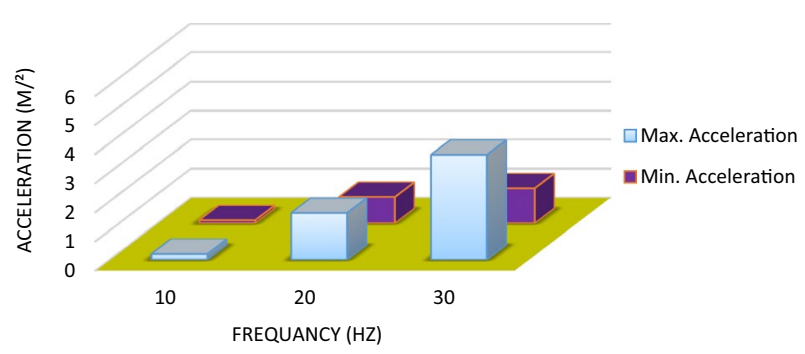

(b)

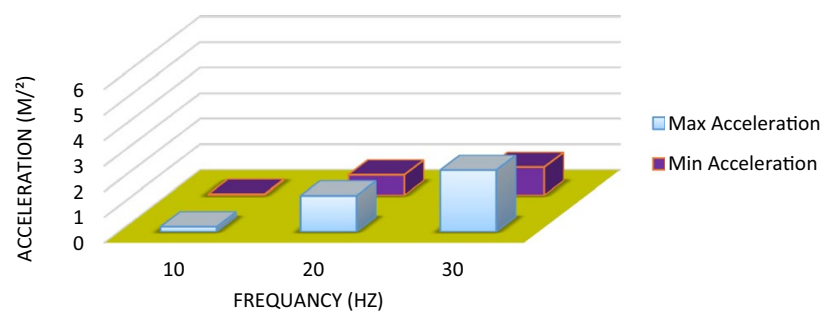

(c)

Fig. 12 The acceleration versus frequency for different spacing (s), a at $S=2 B$, b at $S=4 B, c$ at $S=6 B$ ) for soaked condition

2 bases increases from $2 \mathrm{~B}$ to $4 \mathrm{~B}$, at a frequency of $12 \mathrm{~Hz}$, $23 \%$ in the case of dry, and $32.9 \%$ in the case of soaking. When the gap increases from $2 \mathrm{~B}$ to $6 \mathrm{~B}$, the reduction is $47 \%$ and $65.8 \%$ in dry and soaking conditions, respectively. At a frequency of $18 \mathrm{~Hz}$, the acceleration value decreased (9.1\% and $10.91 \%)$ in dry and soaking conditions, respectively, when the gap increased from $2 \mathrm{~B}$ to $6 \mathrm{~B}$, and decreased $(28.1 \%$ and $23 \%)$ in the case of dry and soaking, respectively, when the gap increased from $2 \mathrm{~B}$ to $6 \mathrm{~B}$. For a frequency of $24 \mathrm{~Hz}$, the reduction is $11.16 \%$ in the case of dehydration and $13.94 \%$ in the case of soaking when the distances increase from $2 B$ to $4 B$, and when the gap increases from $2 B$ to $6 B$, the quantity of acceleration decreases by $37 \%$ and $43 \%$ within the case of dryness and soaking, respectively.

- Reduced acceleration in soaking when spacing increases from $2 \mathrm{~B}$ to $4 \mathrm{~B}$ or 6B greater than the decrease in dry volume acceleration for the same spacing.

Acknowledgements I would like to express deep gratitude and appreciation and thanks are also extended to the all staff of Civil engineering department, and the staff of Soil Mechanics Laboratory, Diyala University, Iraq.

\section{Compliance with ethical standards}

Conflict of interest The authors declare no conflict of interest.

\section{References}

1. Gazetas G (1991) Formulas and charts for impedances of surface and embedded foundations. J Geotech Eng 117(9):1363-1381. https://doi.org/10.1061/ (Asce)0733-9410(1991)117:9(1363)

2. Rao KNSV (1998) Vibration analysis and foundation dynamics. Wheeler, New Delhi

3. Rao KNSV (2010) Foundation design. Wiley, Hoboken. https:// doi.org/10.1002/9780470825365

4. Tham LG, Qian J, Cheung YK (1998) Dynamic response of a group of flexible foundations to incident seismic waves. Soil Dyn Earthq Eng 17(2):127-137. https://doi.org/10.1016/s0267 -7261(97)00021-3

5. Priyanka G, Kumari R (2012) Seismic interference of two nearby horizontal strip anchors in layered soil. Natl Hazards 63(2):789804. https://doi.org/10.1007/s11069-012-0187-4

6. Priyanka G (2012) FLAC based numerical studies on dynamic interference of two nearby embedded machine foundations. Geotech Geol Eng 30(5):1161-1181. https://doi.org/10.1007/ s10706-012-9530-5

7. Razouki S, Al-Omari R, Nashat IH, Razouki HF, Khalid S (1994) The problem of gypsiferous soils in Iraq. In: Proceeding of the symposium on gypsiferous soils and their effect on structures, NCCL, pp 7-33

8. Nashat IH (1990) Engineering characteristics of some gypseous soils in Iraq. Unpublished Ph.D. thesis. University of Baghdad

9. Chen C (2015) Dynamic interaction between rigid foundations on multi-layered stratum. J Earthq Eng 20(4):505-534. https:// doi.org/10.1080/13632469.2015.1104746

10. Abhijeet S, Ghosh P (2016) Experimental study on dynamic interference effect of two closely spaced machine foundations. Can Geotech J 53(2):196-209. https://doi.org/10.1139/ cgj-2014-0462

11. Chen C (2016) Dynamic interaction between rigid surface foundations on multi-layered half space. Int J Struct Stab Dyn 16(05):1550004. https://doi.org/10.1142/s0219455415500042

12. Sbartai $S$ (2016) Dynamic interaction of two adjacent foundations embedded in a viscoelastic soil. Int J Struct Stab Dyn 16(03):1450110. https://doi.org/10.1142/s0219455414501107

13. Suraparb K, Senjuntichai T (2017) “Dynamic response of two rigid foundations on multi-layered poroelastic medium. IOP Conf Ser Mater Sci Eng 269:012047. https://doi.org/10.1088/1757899x/269/1/012047

14. Han Z, Lin G, Jianbo L (2017) Dynamic 3D foundation-soilfoundation interaction on stratified soil. Int J Struct Stab Dyn 17(03):1750032. https://doi.org/10.1142/s0219455417500328

15. Ali OS, Mahamid M (2018) Dynamic response of a four-cylinder compressor foundation considering the effect of soilfoundation interaction: case study. Struct Congr. https://doi. org/10.1061/9780784481332.047

16. Vicencio F, Nicholas A (2018) Dynamic interaction between adjacent buildings through nonlinear soil during earthquakes. Soil Dyn Earthq Eng 108:130-141. https://doi.org/10.1016/j.soild yn.2017.11.031 
17. Andersen LV (2018) Dynamic soil-structure interaction of polypod foundations. Comput Struct. https://doi.org/10.1016/j. compstruc.2018.07.007

18. Keawsawasvong S, Senjuntichai T, Plangmal R, Kaewjuea W (2019) Rocking vibrations of rigid foundations on multi-layered poroelastic media. Mar Georesour Geotechnol. https://doi. org/10.1080/1064119x.2019.1597229

19. Fattah MY, Al-Mosawi MJ, Al-Ameri AF (2016) Vibration response of saturated sand-foundation system. Earthq Struct 11(1):83107. https://doi.org/10.12989/eas.2016.11.1.083

20. Falah $\mathrm{H}$, Ahmed S (2018) Dynamic response of machine foundation resting on sand-granulated tire rubber mixtures. In: Al-Attar TS, Al-Neami MA, Abdul Sahib WS (eds) MATEC web of conferences, vol 162, p 01021. Doi: 10.1051/matecconf/201816201021

21. Pradhan PK, Mandal A, Baidya DK, Ghosh DP (2008) Dynamic response of machine foundation on layered soil: cone model versus experiments. Geotech Geol Eng 26(4):453-468. https:// doi.org/10.1007/s10706-008-9181-8
22. Carl W, Larsson S (2013) Small-scale testing of frequencydependent compaction of sand using a vertically vibrating plate. Geotech Test J 36(3):20120183. https://doi.org/10.1520/ gtj20120183

23. Fattah MY, Al-Mosawi MJ, Al-Ameri AF (2016) Dynamic response of saturated soil foundation system acted upon by vibration. J Earthq Eng 21(7):1158-1188. https://doi.org/10.1080/13632 469.2016.1210060

24. Zakaria WA, Aljanabi QA, Ibrahim OM (2017) Effect of vibration load on leaching process for sandy gypseous soil. Electron $J$ Geotech Eng 22:12

Publisher's Note Springer Nature remains neutral with regard to jurisdictional claims in published maps and institutional affiliations. 